\section{§46. Mode Locking Phenomena Observed near Stability Boundary of Ideal Interchange Mode of LHD}

Takemura, Y. (Grad. Univ. Advanced Studies), Sakakibara, S., Narushima, Y.

In the Large Helical Device (LHD), non-rotating (NR) $m / n=1 / 1$ mode has been observed in weak magnetic shear regime ${ }^{1,2)}$, where the $m$ and $n$ is poloidal and toroidal mode number, respectively. When the magnetic shear is decreased by controlling external coils and/or driving plasma currents, the NR mode abruptly grow during a discharge and extends the flattening of pressure profiles. The mode appears at a specific position. The error field was measured with an electron beam mapping, and it shows that the error field strength is about $0.06 \%$ to operational toroidal field and produces $m / n=1 / 1$ magnetic island ${ }^{3)}$. The position of O-point is roughly consistent with that of the observed NR mode.

This paper focuses on the precursor of the NR mode in the weak magnetic shear operation. The appearance of the precursor was unclear in previous experiments.

The LHD is a heliotron device with a pair of helical coils and three pair of poloidal coils. All coils are superconductive. The typical major and minor radii in the standard configuration are $3.6 \mathrm{~m}$ and $0.65 \mathrm{~m}$, respectively. In the experiments, we selected the configuration with the magnetic axis, $R_{\mathrm{ax}}$, of $3.6 \mathrm{~m}$, toroidal field at $R_{\mathrm{ax}}, B_{\mathrm{t}}$, of $1.2663 \mathrm{~T}$. The plasma aspect ratio, $A_{\mathrm{p}}$, is set at 7.1. In this configuration in vacuum, central and edge rotational transforms, $1(0) / 2 \pi$ and $\mathrm{l}(\mathrm{a}) / 2 \pi$, are 0.57 and 1.61 , respectively ${ }^{1)}$. The LHD is equipped with ten pairs of resonant magnetic perturbation (RMP) coils. The normalized RMP current of $0.12 \mathrm{kA} / \mathrm{T}$ was applied to reduce the error field. The dominant Fourier component is $m / n=1 / 1$. Two co- neutral beams were applied here to drive plasma current increasing $\mathrm{l}(0) / 2 \pi$. The total port-through power was about $8 \mathrm{MW}$.

The $m / n=1 / 1$ mode frequency slowed down and completely stopped at $4.382 \mathrm{~s}$ as shown in Fig.1 (b). The $m / n=1 / 1$ component started to grow then. Fig.1 (d) shows temporal change of the toroidal angle where the O-point is located at the midplane of the low field side, $\phi_{\mathrm{O}-\text {-point }}$. It shows that the mode rotates in the electron diamagnetic direction, whereas the rotation velocity is not constant. The mode frequency was slowed down with an acceleration and a deceleration, and the deceleration was initiated at $\phi_{\mathrm{O}-\text { point }}=$ $90 \sim 140$ degrees. Finally the mode stopped at $\phi_{\text {O-point }} \sim 140$ degrees at $4.5 \mathrm{~s}$.

Figure 2 indicates the radial structure of soft X-ray (SX) fluctuation before the stop of the mode rotation. The intensity of the fluctuation with the same frequency as that of $m / n=1 / 1$ mode has a peak at normalized minor radius, $r / a \sim 0.68$. The phase difference inside and outside the peak is almost constant as shown in Fig.2 (b). The fact that there is no inversion around the resonance means that the mode has an interchange type structure, not magnetic island like tearing mode.

In summary, the mode locking phenomena have been firstly observed in the weak magnetic shear configuration of LHD. The rotating $m / n=1 / 1$ mode appeared at the beginning of the discharge, the rotation slowed down and stopped when the magnetic shear was decreased by beamdriven plasma current. The amplitude of the mode increased with extension of profile flattening around the resonance when the rotation slowed down, and the mode abruptly grew just after the stop of the rotation. The mode has the radial structure with no inversion around the resonance, which means that the mode has interchange-type structure before the minor collapse.

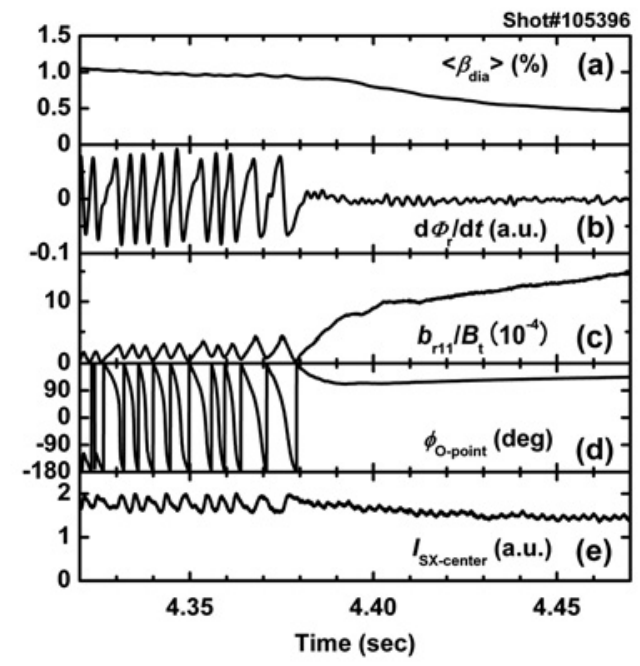

Fig.1 Typical discharge with mode locking. (a) volume averaged beta value, (b) differential signals between two saddle coils separated by $180^{\circ}$, (c) the $m / n=1 / 2$ component of radial magnetic field, (d) toroidal angle where O-point of the mode is located at the midplane of the low field side, and (e) the soft X-ray radiation by line of sight through the plasma core region.

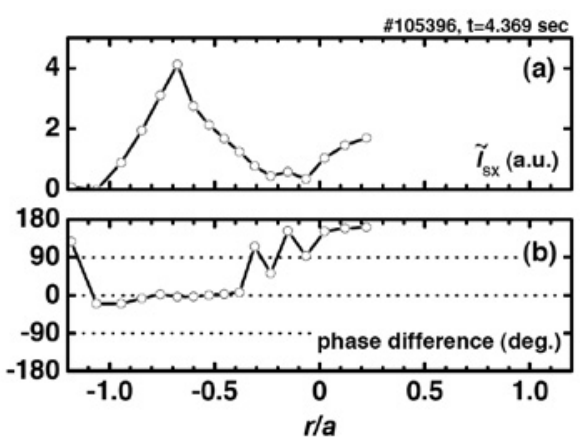

Fig.2 Radial structure of (a) $m / n=1 / 1$ component and (b) phase difference measured with soft $\mathrm{X}$ ray radiation.

1) Sakakibara, S. et al. : Fusion Sci. Technol. 50 (2006) 177.

2) Sakakibara, S. et al. : Fusion Sci. Technol. 58 (2010) 176.

3) Morisaki, T. et al. : Fusion Sci. Technol. 50 (2010) 465. 